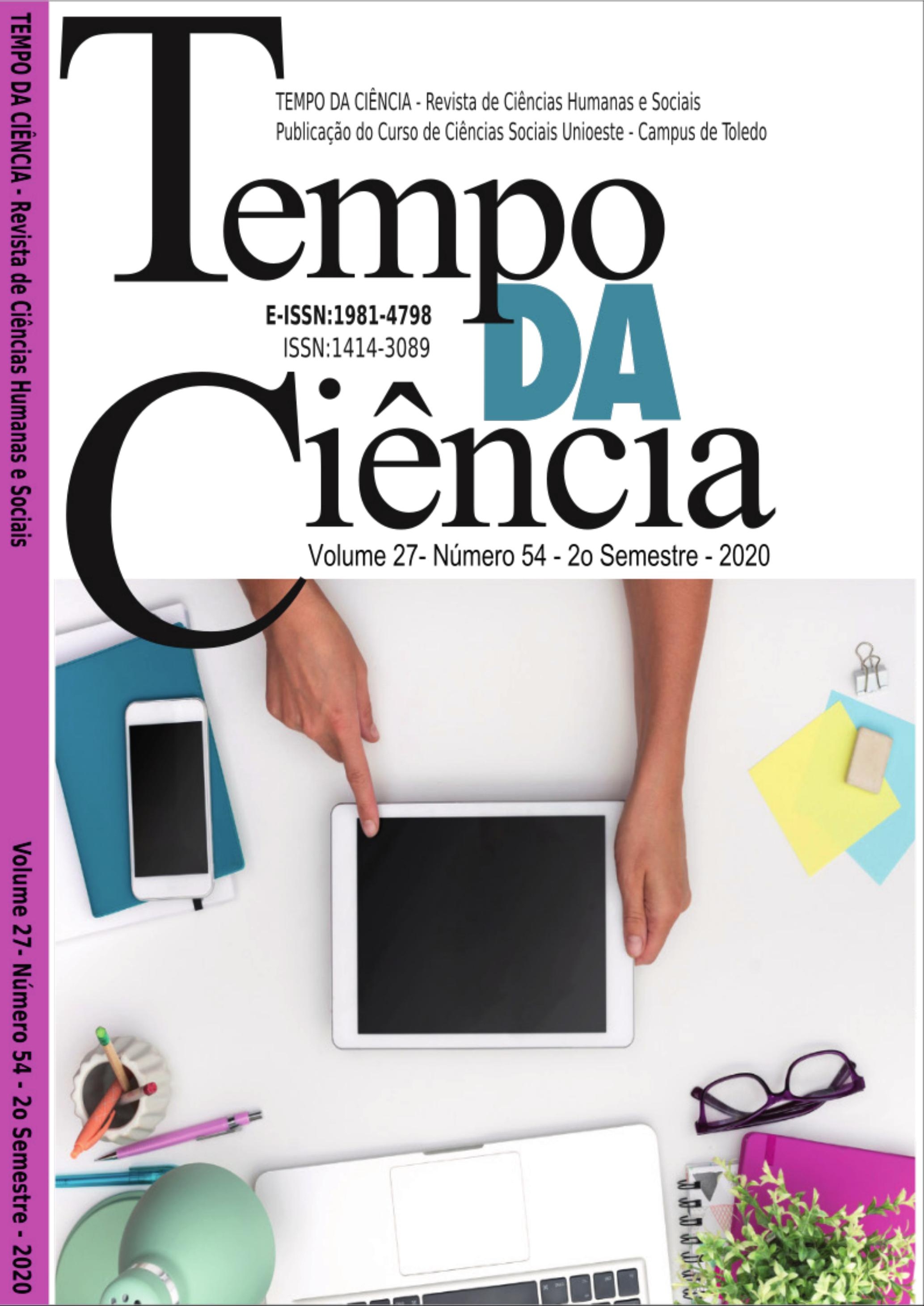




\section{Tempo da Ciência}

Volume 27 Número 54

$2^{\circ}$ semestre de 2020
E-ISSN 1981-4798

ISSN 1414-3089

INDEXADORES:

SISTEMA LATINDEX

SUMÁRIOS DE REVISTAS BRASILEIRAS

Publicação do Curso de Pós-Graduação em Ciências Sociais MESTRADO DA UNIOESTE

CAMPUS DE TOLEDO 
UNIVERSIDADE ESTADUAL DO OESTE DO PARANÁ-UNIOESTE

\author{
REITOR \\ Paulo Sério Wolff \\ DIRETOR DO CAMPUS DE TOLEDO \\ Remi Schorn \\ DIRETOR DO CCHS - CENTRO DE CIÊNCIAS HUMANAS \\ E SOCIAIS CAMPUS DE TOLEDO \\ César Augusto Battisti \\ COORDENADOR DO CURSO DE CIÊNCIAS SOCIAIS \\ Paulo Henrique B. Dias
}

T288 Tempo da Ciência: revista de ciências sociais e humanas / Centro de Ciências Humanas e Sociais da UNIOESTE, Campus de Toledo. Revista de Ciências Sociais do Programa de pós-graduação em Ciências Sociais - mestrado da Unioeste / campus de Toledo- v. 1, n. 1 (1994), -- Toledo : Ed. Toledo, 1994.

Semestral

E-ISSN: 1981-4798

ISSN: 1414-3089

1 Ciências Sociais - Periódicos 2. Ciências Humanas - Periódicos I. Universidade Estadual do Oeste do ParanáCampus de Toledo II. Revista de Ciências Sociais do Programa de pós-graduação em Ciências Sociais - mestrado da Unioeste / campus de Toledo

Revisão ortográfica e gramatical:

Irene Guimarães

Capa:

Vania Sandeleia Vaz da Silva

Diagramação

Fabricio Trindade Ferreira

Suporte técnico:

Paulo Konzen

Endereço para correspondência

UNIOESTE - Núcleo de Documentação, Informação e Pesquisa - NDP

Rua da Faculdade, 645 - Jardim La Salle - Toledo - PR CEP 85903-000

e-mail: revistatempodaciencia@yahoo.com.br 


\section{EDITORA}

Vania Sandeleia Vaz da Silva

\section{COMISSÃO EDITORIAL}

Andréia Vicente da Silva

Gustavo Biasoli Alves

Marco Antonio Arantes

Osmir Dombrowski

Paulo Henrique B. Dias

\section{CONSELHO ASSESSOR CIENTÍFICO}

Adriano Premebida - FADB

Alberto Paulo Neto - PUC-PR

Allan de Paula Oliveira - NESPAR

Ana Cleide Chiarotti Cesário - UEL

Carla Cecília Rodrigues Almeida - UEM

Celso Antonio Fávero - UNEB

Emilce Beatriz Cammarata - NM, Argentina,

Eric Gustavo Cardin - UNIOESTE

Eric Sabourin - CIRAD, França

Erneldo Schallenberger - UNIOESTE

Evaldo Mendes da Silva - UFAL

Gabriel Feltran - UFSCAR

Geraldo Magella Neres - UNIOESTE

Ileizi Luciana Fiorelli Silva - UEL

Joana Coutinho - UFMA

João Virgílio Tagliavini - UFSCar

José Lindomar C. Albuquerque - UNIFESP

Juan Carlos Arriaga- Rodríguez - Universidad de 'Quintana Roo - México

María Lois - Universidad Complutense de Madrid

Maria Salete Souza de Amorim - UFBA

Martha C. Ramirez-Galvez - UEL

Miguel Angelo Lazzaretti - UNIOESTE

Oscar Calavia Sáez - UFSC

Otávio Velho - UFRJ

Paulo Roberto Azevedo - UNIOESTE

Rafael Antônio Duarte Villa - USP

René E. Gertz - PUCRS e UFRGS

Ricardo Cid Fernandes - UFPR

Rosana Kátia Nazzari - UNIOESTE

Senilde Guanaes - UNILA

Wagner Pralon Mancuso - USP

Yonissa Marmitt Wadi - UNIOESTE 


\section{Sumário:}

Apresentação: Publicação de artigos escritos por mulheres na revista Tempo da Ciência nos últimos 10 anos Vania Sandeleia Var, da Silva Pg 6-10

Reestruturação do serviço público na era do trabalho flexível Lícia Helena de Oliveira Medeiros, Paulo Gracino Junior

$$
\operatorname{Pg} 11-24
$$

A construção do conceito de caboclo como demarcador social de inferioridade no sul do Brasil

Taiza Gabriela Zanatta Crestani, Silvio Antonio Colognese, Paulo Ricardo Bavaresco

$$
\operatorname{Pg} 25-37
$$

Breve ensaio sobre linhas, conflitos e transversais

Douglas Ferreira Gadelha Campelo

$$
\operatorname{Pg} 3849
$$

Cantos que aprendemos nos sonhos: os grupos de canto/corais Ava-guarani e a transmissão de saberes através da experiência onírica na aldeia Ocoy/ Paraná.

$$
\begin{gathered}
\text { Denize Refatti } \\
\text { Pg 50-59 }
\end{gathered}
$$

John Rawls e Charles Taylor: Liberalismo igualitário e comunitarismo/multiculturalismo. Um diálogo possível?

$$
\begin{gathered}
\text { Rosangela da Silva, Francioli Bagatin } \\
\text { Pg 60-72 }
\end{gathered}
$$

As classes sociais no Brasil: uma proposta de leitura relacional

$$
\begin{gathered}
\text { Alexandre Aparecido dos Santos } \\
\text { Pg } 73-85
\end{gathered}
$$

A Redução de Danos como estratégia de promoção de orientação e cuidados para usuários de drogas: uma revisão sobre experiências de alguns países.

$$
\begin{aligned}
& \text { Jaqueline de Sousa Gomes, Nalayne Mendonça Pinto } \\
& \qquad \operatorname{Pg} 86-102
\end{aligned}
$$

Mulheres feministas e evangélicas: a construção racional de uma nova identidade a partir dos estudos de gênero e do acesso ao ensino superior Milena Geisa Dos Santos Martins

Pg 103-116

As deputadas federais eleitas por São Paulo, em 2018, são feministas ou antifeministas?

Isadora Gonzaga Postingher, Vania Sandeleia Vaz da Silva

Pg 117-132

Resenha: Os Brasis de Antonio Lino em Branco Vivo.

Marco Antonio Arantes

Pg 133-136 